\title{
Community Health Workers' Augment The Cascade of TB Detection To Care In Urban Slums of Two Metro Cities In India
}

\author{
Rajaram Subramanian Potty ( $\sim$ rajaram@khpt.org ) \\ Karnataka Health Promotion Trust https://orcid.org/0000-0002-1232-143X
}

\section{Karthikeyan K}

Karnataka Health Promotion Trust

\section{Rajesham A}

Office of the Joint Director (TB), Hyderabad

Ramesh Chandra Reddy

Office of the Joint Director (TB), Bengaluru

\section{Anil Singarajipura}

Office of the Joint Director (TB), Bengaluru

\section{Poornima Bathi Siddappa}

Karnataka Health Promotion Trust

\section{Prarthana BS}

Karnataka Health Promotion Trust

\section{Raghavendra T}

Karnataka Health Promotion Trust

\section{Mohan HL}

Karnataka Health Promotion Trust

\section{Reuben Swamickan}

USAID India

Amar Shah

USAID India

\section{Vikas Panibatla}

TB Alert India

\section{Ramesh Dasari}

TB Alert India

\section{Reynold Washington}

Karnataka Health Promotion Trust

\section{Research Article}

Keywords: Community health workers, Tuberculosis, Hyderabad, Bengaluru, Successful treatment outcome, Slum areas, Community engagement

Posted Date: February 4th, 2021

DOI: https://doi.org/10.21203/rs.3.rs-159862/v1

License: (a) (i) This work is licensed under a Creative Commons Attribution 4.0 International License. Read Full License 


\section{Abstract}

\section{Background:}

Tuberculosis Health Action Learning Initiative (THALI) funded by USAID is a person-centered initiative, supporting vulnerable urban populations to gain access to TB services. THALI trained and placed 112 Community health workers (CHWs) to detect and support individuals with TB symptoms or disease within urban slums in two cities, Hyderabad and Bengaluru, covering a population of about 3 Million.

\section{Method:}

$\mathrm{CHWs}$ visited the slums once in a fortnight. They conducted TB awareness activities. They referred individuals with TB symptoms for sputum testing to nearest public sector labs. They visited those testing TB positive, once a fortnight in the intensive phase, and once a month thereafter. They supported TB patients and families with counselling, contact screening and social scheme linkages. They complemented the shortfall in urban TB government field staff numbers and their capacity to engage with TB patients. Data on CHWs' patient referral for TB diagnosis and treatment support activities was entered into a data-base and analyzed to examine CHWs' role in the cascade of TB care. We compared achievements of six monthly referral cohorts from September 2016 to February 2019.

\section{Results:}

Overall, 31617 (approximately 1\%) of slum population were identified as TB symptomatic and referred for diagnosis. Among the referred persons, 23976 (76\%) underwent testing of which 3841 (16\%) were TB positive. Overall, $3812(99 \%)$ were initiated on treatment and 2760(72\%) agreed for regular followed up by CHWs.

Fifty-seven percent of 2952 referred were tested in the first cohort, against $86 \%$ of 8315 in the last cohort. The annualized case detection rate through $\mathrm{CHW}$ referrals in Bengaluru increased from 5.5 to 52.0 per 100000 during the period, while in Hyderabad it was 35.4 initially and increased up to 118.9 per 100000 persons. The treatment success rate was $87.1 \%$ among 193 in the first cohort versus $91.3 \%$ among 677 in the last cohort.

\section{Conclusions}

CHWs in urban slums augment TB detection to care cascade. Their performance and TB treatment outcomes improve over time. It would be important to examine the cost per TB case detected and successfully treated.

\section{Introduction}

India continues to contribute greatly to the global tuberculosis (TB) burden due to its large population size and high TB incidence. The Global TB report 2020 indicated that India was listed as one of 30 high TB burden countries and accounted for $26 \%$ of the TB cases globally [1]. India also accounts for $25 \%$ of total estimated missing cases globally, a large gap between the estimated and reported number of new TB cases [2]. Many factors contribute to this scenario. About half of TB symptomatic persons first seek care within the private sector which is not yet fully engaged in TB control [3]. Under-reporting of TB patients as a result of system constraints or attitudes of health personnel are other reasons for this gap [4-11]. In order to close this gap in TB detection, intensified efforts are required especially among populations who are more vulnerable to TB and who have challenges in accessing TB diagnostic services [2]. Vulnerable populations with TB symptoms need knowledge and access to TB diagnostic and treatment facilities in order to detect the disease in a timely manner.

Early diagnosis and treatment for TB reduces the risk of disease transmission from one individual to others in the family or in close contact [12]. Early initiation of treatment also improves TB treatment outcomes and reduces the complications as well as the out of pocket expenditure incurred by individuals and their families $[13,14]$. The two well-known pathways to early diagnosis of TB are the patient-initiated pathway and the screening-pathway [15]. In the patient-initiated pathway, the person with TB actively seeks care from the health care provider when he or she experiences symptoms. In the screening pathway, individuals 
are screened for symptoms suggestive of TB and the persons with TB suggestive symptoms are referred for appropriate TB testing. The latter approach has the potential advantage of diagnosing TB in persons who would have otherwise missed or delayed their diagnosis [15]. However, in order to be cost-efficient with this approach, the focus must be among populations that are at a greater risk of developing TB disease. Among others, these include individuals living in urban slums, individuals who are exposed to occupational environments that compromise lung function, individuals who are severely malnourished, and persons living with HIV [16-20].

The United States Agency for International Development/India (USAID/India) awarded a grant namely 'Tuberculosis Health Action Learning Initiative (THALI)' to a consortium of partners led by Karnataka Health Promotion Trust (KHPT), Bengaluru. KHPT works in close partnership with TB Alert India (TBAI) in Hyderabad, and in collaboration with the Revised National Tuberculosis Control program (RNTCP) now referred to as the National TB Elimination Program (NTEP). The THALI project is a patient centered, family-focused TB prevention and care initiative supporting vulnerable people to gain access to quality TB care services from health care providers of the patient's choice. One of the strategies adopted by THALI was to enhance community outreach and engagement within vulnerable communities through a dedicated cadre of community health workers (CHWs). This was necessary, considering the $30-50 \%$ gap in recruitment of TB health visitors (TBHV) in metro-cities and large towns, the front-line work force within the NTEP. As well, the TB case load within these high TB prevalent communities' demands additions to the work-force in order to be able to support TB patients with counselling for treatment adherence and linkages to nutrition and other social support schemes, that often remain inaccessible to these populations. Shortfalls in the number and capacity of front-line health care workers weakens the last mile in the delivery of TB care services. Innovative strategies such as development of alternative cadres and task shifting are attempts to address these shortfalls [20-21]. A systematic analysis of the program learnings from community engagement activities that are conducted through $\mathrm{CHWs}$ is important to contribute to evidence based policy and program related decision-making [22]. In this paper we systematically analyze and describe the potential augmentation in the TB care cascade achieved through the community engagement activities conducted in two large metro cities in India, namely Bengaluru and Hyderabad. The uniqueness of this paper is the depiction of the entire continuum of care of TB patients through community engagement.

\section{Methods}

\section{Study setting}

The project area included urban slum areas of Hyderabad and Bengaluru cities. We identified and mapped 647 slums in Bengaluru city and 942 slums in Hyderabad city, catering to about 323,000 households and 1.5 million population covered by 24 TB units (TUs) in Bengaluru and about 294,000 households and 1.45 million population covered by 19 TUs in Hyderabad.

\section{Community Engagement Strategy}

The THALI project trained and placed $112 \mathrm{CHWs}$ to support individuals with TB symptoms or confirmed disease living within urban slums in the two cities, Hyderabad and Bengaluru. Each $\mathrm{CHW}$ covered about 5-25 slum areas with a population ranging from 20000-32000 and aligned to the geographic coverage by the Designated Microscopy Centre (DMC), a government health care facility that conducts sputum microscopy and onward referral for CBNAAT testing (Cartridge Based Nucleic Acid Amplification Test) for TB diagnosis. 14 Community Coordinators (CC) supervised and supported about 8-10 CHWs, each. CHWs conducted Information Education and Communication (IEC) campaigns on TB within the community through in-person contacts, small group meetings, large group meetings and school-based education programs and were involved in the periodic active case finding campaigns that were conducted by the government. CHWs visited each slum area once in a fortnight.

Using a 'screening pathway', CHWs actively identified individuals with TB suggestive symptoms during the community engagement activities listed above. Any person who had persistent cough for more than 14 days and/or had night sweats, sudden weight loss, blood in sputum, reduced appetite, persistent chest pain and enlarged lumps (lymph nodes) was identified as a TB symptomatic. They referred TB symptomatics for sputum testing to the DMC. The referral process included collecting 
socio-demographic details, filling up a form in triplicate and handing over two sputum cups, with clear instructions on how to collect an on-the-spot sample and an early morning sputum sample. If the individual could not go themselves to the DMC to hand over the samples for testing, the $\mathrm{CHW}$ would transport the sample for testing on behalf of the referred person, adhering to standard infection prevention guidelines that they were trained in. Whenever a sample tested positive for TB, the CHW would accompany that individual to the public health facility for counselling and treatment initiation. Following initiation of the TB treatment, with verbal recorded consent of the individual, the $\mathrm{CHW}$ followed up the person twice a month during the intensive phase and once a month during the continuation phase of treatment. During the follow-up visits, the CHW would mobilize family level support, provide counselling support and monitor treatment adherence, give nutritional advice and support the individual to obtain social entitlements, including enrolment into the Government's direct benefit transfer (DBT) scheme of Indian rupees 500 per month. Often, these patients were supported to open a bank account and link their Aadhar (Unique Identification Number) and telephone number to the bank account, in order to avail the DBT. Many patients were not readily willing to share these details, as they feared misuse of the data. TB patients and family level treatment supporters were also motivated to attend patient support group (PSG) meetings. The patient's weight gain was monitored during follow-up visits. Patients were reminded about and referred for follow-up tests, counselled on relevant behavior change (smoking and alcohol consumption) and referred to a doctor for management of adverse drug reactions or for side effects management and co-morbidities.

\section{Data Collection}

All counselling inputs by the $\mathrm{CHW}$, weight measurements, follow-up test results and treatment adherence were documented by the $\mathrm{CHW}$, under the supportive supervision of the CC, on a Patient Referral and Diagnosis (PRAD) card form during referral and a Prevention, Care and Support card (PCS) during treatment adherence support. The outcome of the TB treatment was recorded and validated by project medical personnel and the NTEP Senior TB Treatment Supervisor (STS). Both forms once filled were verified for completeness by the CC, before entry into the computerized management information system (CMIS) on a regular basis.

\section{Data analysis}

We analyzed both the PRAD and the PCS data collected by the CHW. In order to examine the trends in the TB care cascade, we grouped the referred patients into various cohorts according to the referral months and year. We made five cohorts each of six months' duration based on the months and year of referral, starting in September 1, 2016 and ending as of February $29,2019$. We examined the number of referrals, the proportion of persons who had undergone the tests, the proportion among them diagnosed as TB either microbiologically or clinically, and proportion started on anti-tuberculosis treatment (ATT). For each of these cohorts, we examined the percentage of patients with successful treatment outcomes, that is who are either declared cured of TB or completed the course of TB treatment. In addition, we also examined successful treatment outcomes according to socio-economic and demographic characteristics, number of follow-up visits, whether missed any dose during the treatment, and type of action taken by the CHW during the follow-up visit. We applied the multiple logistic regression model to identify the likelihood of successful treatment outcome across the referral cohorts. We adopted stepwise backward logistic regression models to explore significant changes in the successful treatment outcomes over the time periods. If the p-value of any group as a whole was more than 0.10 , such variables were eliminated from the multiple logistic regression. We constructed three different logistic regression models that included the number of follow-up visits during the intensive phase, the number of follow-up visits during the continuation phase and the total number of follow-up visits in addition to the various characteristics mentioned. We applied three different models for these three visits separately in order to understand the overall change in the treatment outcome across various referral cohorts, after controlling for these visits along with other potential factors. It is also possible that these visits can be potentially interrelated and we thus chose not to use these three visits in the same model.

\section{Ethical approval}

The Institutional Ethics Committee of St John's Medical College and Hospital provided the ethics approval for program data review and analysis. The State TB office and local NTEP officials in the two states provided regulatory approval for access to 
Nikshay data and to interview patients and conduct follow-up visits.

\section{Results}

Overall, from September 2016 to February 2019, the CHWs in Bengaluru and Hyderabad had referred 31617 TB symptomatic individuals for TB diagnoses (approximately $1 \%$ of the total slum population). Of those referred, 23976 (76\%) persons had completed TB testing and were provided with results (see Table 1). The proportion of referred individuals undergoing testing increased over time from $57 \%$ in the first cohort to $86 \%$ in the last. This increase to over $80 \%$ of those referred being tested and receiving results was seen in both cities, across gender and all age groups. The annualized case detection rate through $\mathrm{CHW}$ referrals in Bengaluru increased from 5.5 to 52.0 per 100000 during the period, while in Hyderabad it was 35.4 initially and increased up to 118.9 per 100000 persons. 
Table 1

Coverage indicators reflecting the cascade of TB care including number of persons referred by $\mathrm{CHW}$, undergone test, tested positive, started treatment, followed-up according to various referral cohorts

\begin{tabular}{|c|c|c|c|c|c|c|c|c|c|}
\hline $\begin{array}{l}\text { Six } \\
\text { monthly } \\
\text { referral } \\
\text { cohorts }\end{array}$ & $\begin{array}{l}\text { Number } \\
\text { referred }\end{array}$ & $\begin{array}{l}\text { Number } \\
\text { tested }\end{array}$ & $\begin{array}{l}\text { Number } \\
\text { tested } \\
\text { positive }\end{array}$ & $\begin{array}{l}\text { Number } \\
\text { started } \\
\text { treatment }\end{array}$ & $\begin{array}{l}\text { Registered } \\
\text { for follow- } \\
\text { up by } \\
\text { CHW }\end{array}$ & $\begin{array}{l}\% \\
\text { undergone } \\
\text { test }\end{array}$ & $\begin{array}{l}\% \\
\text { tested } \\
\text { positive }\end{array}$ & $\begin{array}{l}\% \text { started } \\
\text { treatment }\end{array}$ & $\begin{array}{l}\% \\
\text { Registered } \\
\text { for follow- } \\
\text { up }\end{array}$ \\
\hline \multicolumn{10}{|l|}{ Total } \\
\hline $\begin{array}{l}\text { Sep } 2016 \text { - } \\
\text { Feb, } 2017\end{array}$ & 2952 & 1691 & 298 & 296 & 203 & 57.3 & 17.6 & 99.3 & 68.6 \\
\hline $\begin{array}{l}\text { Mar - Aug, } \\
2017\end{array}$ & 4176 & 2525 & 516 & 515 & 362 & 60.5 & 20.4 & 99.8 & 70.3 \\
\hline $\begin{array}{l}\text { Sep, } 2017 \\
\text {-Feb, } 2018\end{array}$ & 8270 & 6077 & 1,082 & 1,079 & 800 & 73.5 & 17.8 & 99.7 & 74.1 \\
\hline $\begin{array}{l}\text { Mar -Aug, } \\
2018\end{array}$ & 7904 & 6521 & 1,022 & 1,010 & 696 & 82.5 & 15.7 & 98.8 & 68.9 \\
\hline $\begin{array}{l}\text { Sep, } 2018 \\
\text { - Feb } 2019\end{array}$ & 8315 & 7162 & 923 & 912 & 699 & 86.1 & 12.9 & 98.8 & 76.6 \\
\hline \multicolumn{10}{|l|}{ Bengaluru } \\
\hline $\begin{array}{l}\text { Sep } 2016 \text { - } \\
\text { Feb, } 2017\end{array}$ & 1179 & 588 & 41 & 40 & 31 & 49.9 & 7.0 & 97.6 & 77.5 \\
\hline $\begin{array}{l}\text { Mar - Aug, } \\
2017\end{array}$ & 1946 & 1118 & 105 & 104 & 85 & 57.5 & 9.4 & 99.0 & 81.7 \\
\hline $\begin{array}{l}\text { Sep, } 2017 \\
\text {-Feb, } 2018\end{array}$ & 3436 & 2632 & 220 & 217 & 185 & 76.6 & 8.4 & 98.6 & 85.3 \\
\hline $\begin{array}{l}\text { Mar -Aug, } \\
2018\end{array}$ & 3593 & 3159 & 303 & 294 & 226 & 87.9 & 9.6 & 97.0 & 76.9 \\
\hline $\begin{array}{l}\text { Sep, } 2018 \\
\text { - Feb } 2019\end{array}$ & 4978 & 3979 & 393 & 382 & 315 & 79.9 & 9.9 & 97.2 & 82.5 \\
\hline \multicolumn{10}{|l|}{ Hyderabad } \\
\hline $\begin{array}{l}\text { Sep } 2016 \text { - } \\
\text { Feb, } 2017\end{array}$ & 1,773 & 1103 & 257 & 256 & 172 & 62.2 & 23.3 & 99.6 & 67.2 \\
\hline $\begin{array}{l}\text { Mar - Aug, } \\
2017\end{array}$ & 2,230 & 1407 & 411 & 411 & 277 & 63.1 & 29.2 & 100.0 & 67.4 \\
\hline $\begin{array}{l}\text { Sep, } 2017 \\
\text {-Feb, } 2018\end{array}$ & 4,834 & 3445 & 862 & 862 & 615 & 71.3 & 25.0 & 100.0 & 71.3 \\
\hline $\begin{array}{l}\text { Mar -Aug, } \\
2018\end{array}$ & 4,311 & 3362 & 719 & 716 & 470 & 78.0 & 21.4 & 99.6 & 65.6 \\
\hline $\begin{array}{l}\text { Sep, } 2018 \\
\text { - Feb } 2019\end{array}$ & 3337 & 3183 & 530 & 530 & 384 & 95.4 & 16.7 & 100.0 & 72.5 \\
\hline \multicolumn{10}{|l|}{ Male } \\
\hline $\begin{array}{l}\text { Sep } 2016 \text { - } \\
\text { Feb, } 2017\end{array}$ & 1549 & 893 & 170 & 169 & 114 & 57.7 & 19.0 & 99.4 & 67.5 \\
\hline $\begin{array}{l}\text { Mar - Aug, } \\
2017\end{array}$ & 2,142 & 1305 & 290 & 289 & 213 & 60.9 & 22.2 & 99.7 & 73.7 \\
\hline $\begin{array}{l}\text { Sep, } 2017 \\
\text {-Feb, } 2018\end{array}$ & 4,159 & 3067 & 574 & 573 & 429 & 73.7 & 18.7 & 99.8 & 74.9 \\
\hline
\end{tabular}




\begin{tabular}{|c|c|c|c|c|c|c|c|c|c|}
\hline $\begin{array}{l}\text { Six } \\
\text { monthly } \\
\text { referral } \\
\text { cohorts }\end{array}$ & $\begin{array}{l}\text { Number } \\
\text { referred }\end{array}$ & $\begin{array}{l}\text { Number } \\
\text { tested }\end{array}$ & $\begin{array}{l}\text { Number } \\
\text { tested } \\
\text { positive }\end{array}$ & $\begin{array}{l}\text { Number } \\
\text { started } \\
\text { treatment }\end{array}$ & $\begin{array}{l}\text { Registered } \\
\text { for follow- } \\
\text { up by } \\
\text { CHW }\end{array}$ & $\begin{array}{l}\% \\
\text { undergone } \\
\text { test }\end{array}$ & $\begin{array}{l}\% \\
\text { tested } \\
\text { positive }\end{array}$ & $\begin{array}{l}\% \text { started } \\
\text { treatment }\end{array}$ & $\begin{array}{l}\% \\
\text { Registered } \\
\text { for follow- } \\
\text { up }\end{array}$ \\
\hline $\begin{array}{l}\text { Mar -Aug, } \\
2018\end{array}$ & 4,073 & 3361 & 534 & 524 & 360 & 82.5 & 15.9 & 98.1 & 68.7 \\
\hline $\begin{array}{l}\text { Sep, } 2018 \\
\text { - Feb } 2019\end{array}$ & 4286 & 3674 & 513 & 504 & 387 & 85.7 & 14.0 & 98.2 & 76.8 \\
\hline \multicolumn{10}{|l|}{ Female } \\
\hline $\begin{array}{l}\text { Sep } 2016 \text { - } \\
\text { Feb, } 2017\end{array}$ & 1403 & 798 & 128 & 127 & 89 & 56.9 & 16.0 & 99.2 & 70.1 \\
\hline $\begin{array}{l}\text { Mar - Aug, } \\
2017\end{array}$ & 2,034 & 1220 & 226 & 226 & 149 & 60.0 & 18.5 & 100.0 & 65.9 \\
\hline $\begin{array}{l}\text { Sep, } 2017 \\
\text {-Feb, } 2018\end{array}$ & 4,111 & 3010 & 508 & 506 & 371 & 73.2 & 16.9 & 99.6 & 73.3 \\
\hline $\begin{array}{l}\text { Mar -Aug, } \\
2018\end{array}$ & 3,831 & 3160 & 488 & 486 & 336 & 82.5 & 15.4 & 99.6 & 69.1 \\
\hline $\begin{array}{l}\text { Sep, } 2018 \\
\text { - Feb } 2019\end{array}$ & 3766 & 3488 & 410 & 408 & 312 & 92.6 & 11.8 & 99.5 & 76.5 \\
\hline \multicolumn{10}{|l|}{$\begin{array}{l}\text { Age }<15 \\
\text { years }\end{array}$} \\
\hline $\begin{array}{l}\text { Sep } 2016 \text { - } \\
\text { Feb, } 2017\end{array}$ & 185 & 90 & 11 & 11 & 7 & 48.6 & 12.2 & 100.0 & 63.6 \\
\hline $\begin{array}{l}\text { Mar - Aug, } \\
2017\end{array}$ & 334 & 174 & 24 & 24 & 19 & 52.1 & 13.8 & 100.0 & 79.2 \\
\hline $\begin{array}{l}\text { Sep, } 2017 \\
\text {-Feb, } 2018\end{array}$ & 833 & 539 & 55 & 55 & 43 & 64.7 & 10.2 & 100.0 & 78.2 \\
\hline $\begin{array}{l}\text { Mar -Aug, } \\
2018\end{array}$ & 636 & 452 & 68 & 68 & 36 & 71.1 & 15.0 & 100.0 & 52.9 \\
\hline $\begin{array}{l}\text { Sep, } 2018 \\
\text { - Feb } 2019\end{array}$ & 489 & 394 & 32 & 32 & 23 & 80.6 & 8.1 & 100.0 & 71.9 \\
\hline \multicolumn{10}{|l|}{$\begin{array}{l}\text { Age } 15- \\
49 \text { years }\end{array}$} \\
\hline $\begin{array}{l}\text { Sep } 2016 \text { - } \\
\text { Feb, } 2017\end{array}$ & 1,164 & 656 & 224 & 222 & 154 & 56.4 & 34.1 & 99.1 & 69.4 \\
\hline $\begin{array}{l}\text { Mar - Aug, } \\
2017\end{array}$ & 2,367 & 1388 & 391 & 390 & 263 & 58.6 & 28.2 & 99.7 & 67.4 \\
\hline $\begin{array}{l}\text { Sep, } 2017 \\
\text {-Feb, } 2018\end{array}$ & 4,346 & 2972 & 795 & 793 & 580 & 68.4 & 26.7 & 99.7 & 73.1 \\
\hline $\begin{array}{l}\text { Mar -Aug, } \\
2018\end{array}$ & 5,410 & 4435 & 765 & 758 & 529 & 82.0 & 17.2 & 99.1 & 69.8 \\
\hline $\begin{array}{l}\text { Sep, } 2018 \\
\text { - Feb } 2019\end{array}$ & 5576 & 4749 & 707 & 700 & 537 & 85.2 & 14.9 & 99.0 & 76.7 \\
\hline \multicolumn{10}{|l|}{$\begin{array}{l}\text { Age } 50+ \\
\text { years }\end{array}$} \\
\hline $\begin{array}{l}\text { Sep } 2016 \text { - } \\
\text { Feb, } 2017\end{array}$ & 576 & 361 & 63 & 63 & 42 & 62.7 & 17.5 & 100.0 & 66.7 \\
\hline
\end{tabular}




\begin{tabular}{|c|c|c|c|c|c|c|c|c|c|}
\hline $\begin{array}{l}\text { Six } \\
\text { monthly } \\
\text { referral } \\
\text { cohorts }\end{array}$ & $\begin{array}{l}\text { Number } \\
\text { referred }\end{array}$ & $\begin{array}{l}\text { Number } \\
\text { tested }\end{array}$ & $\begin{array}{l}\text { Number } \\
\text { tested } \\
\text { positive }\end{array}$ & $\begin{array}{l}\text { Number } \\
\text { started } \\
\text { treatment }\end{array}$ & $\begin{array}{l}\text { Registered } \\
\text { for follow- } \\
\text { up by } \\
\text { CHW }\end{array}$ & $\begin{array}{l}\% \\
\text { undergone } \\
\text { test }\end{array}$ & $\begin{array}{l}\% \\
\text { tested } \\
\text { positive }\end{array}$ & $\begin{array}{l}\% \text { started } \\
\text { treatment }\end{array}$ & $\begin{array}{l}\% \\
\text { Registered } \\
\text { for follow- } \\
\text { up }\end{array}$ \\
\hline $\begin{array}{l}\text { Mar - Aug, } \\
2017\end{array}$ & 888 & 500 & 101 & 101 & 80 & 56.3 & 20.2 & 100.0 & 79.2 \\
\hline $\begin{array}{l}\text { Sep, } 2017 \\
\text {-Feb, } 2018\end{array}$ & 1,800 & 1309 & 232 & 231 & 177 & 72.7 & 17.7 & 99.6 & 76.6 \\
\hline $\begin{array}{l}\text { Mar -Aug, } \\
2018\end{array}$ & 2,083 & 1691 & 189 & 184 & 131 & 81.2 & 11.2 & 97.4 & 71.2 \\
\hline $\begin{array}{l}\text { Sep, } 2018 \\
\text { - Feb } 2019\end{array}$ & 2299 & 1959 & 184 & 180 & 139 & 85.2 & 9.4 & 97.8 & 77.2 \\
\hline Total & 31617 & 23976 & 3841 & 3812 & 2760 & 75.8 & 16.0 & 99.2 & 72.4 \\
\hline
\end{tabular}

Overall, $16 \%$ of those tested were diagnosed to have TB and the TB detection among those tested reduced from 17.6 to $12.9 \%$. The proportion of people who tested TB positive was consistently higher in Hyderabad than in Bengaluru, among males than among females and among those aged 15-49.

Overall, $99 \%$ of individuals diagnosed with TB were started on ATT with minor fluctuations in levels between Bengaluru and Hyderabad. Overall, $72 \%$ of them consented to be registered and documented for the follow-up visits using the PCS card, with proportions ranging from between $69 \%$ and $77 \%$. The proportion of TB patients who consented to be registered for PCS is comparatively lower in Hyderabad as compared to Bengaluru for all the referral cohorts examined. Also, in few of the cohorts slightly lesser proportion of TB patients aged below 15 years were registered for follow-up visits as compared to TB patients in other age groups.

Table 2 provides the mean and total number of follow-up visits during the intensive and the continued treatment phases. Though, fluctuations were observed in the mean number of follow-up visits with Bengaluru indicating a reduction and Hyderabad showing an increase across various referral cohorts, the standard deviation reduced across groups indicating that the intensity of follow up became more or less uniform over time. We also noticed the same kind of results for the visits in the intensive and continuation phases as well. 
Table 2

Mean number of total follow-up visits, mean number of visits during intensive phase and during continuation phase for TB patients referred by CHW according to different referral cohorts

\begin{tabular}{|c|c|c|c|c|c|c|c|}
\hline \multirow[t]{2}{*}{ Referral cohorts } & \multicolumn{2}{|c|}{$\begin{array}{l}\text { Total follow-up } \\
\text { visits }\end{array}$} & \multicolumn{2}{|c|}{$\begin{array}{l}\text { Follow-up visits at Continuation } \\
\text { phase }\end{array}$} & \multicolumn{2}{|c|}{$\begin{array}{l}\text { Follow-up visits at Intensive } \\
\text { Phase }\end{array}$} & \multirow[t]{2}{*}{$\begin{array}{l}\text { Number of } \\
\text { cases }\end{array}$} \\
\hline & Mean & SD & Mean & SD & Mean & SD & \\
\hline \multicolumn{8}{|l|}{ Total } \\
\hline $\begin{array}{l}\text { Sep } 2016 \text { - Feb, } \\
2017\end{array}$ & 6.2 & 4.2 & 3.8 & 2.8 & 2.4 & 2.0 & 203 \\
\hline Mar - Aug, 2017 & 7.4 & 5.0 & 4.5 & 3.2 & 2.9 & 2.4 & 362 \\
\hline $\begin{array}{l}\text { Sep, } 2017 \text {-Feb, } \\
2018\end{array}$ & 7.5 & 4.2 & 4.4 & 2.7 & 3.0 & 2.1 & 800 \\
\hline Mar -Aug, 2018 & 6.9 & 2.9 & 3.8 & 2.3 & 3.1 & 1.3 & 696 \\
\hline $\begin{array}{l}\text { Sep, } 2018 \text { - Feb } \\
2019\end{array}$ & 8.4 & 3.6 & 5.0 & 3.1 & 3.4 & 1.2 & 699 \\
\hline \multicolumn{8}{|l|}{ Bengaluru } \\
\hline $\begin{array}{l}\text { Sep } 2016 \text { - Feb, } \\
2017\end{array}$ & 11.0 & 6.8 & 5.8 & 4.7 & 5.2 & 2.9 & 31 \\
\hline Mar - Aug, 2017 & 12.5 & 7.0 & 6.8 & 4.8 & 5.6 & 2.9 & 85 \\
\hline $\begin{array}{l}\text { Sep, } 2017 \text {-Feb, } \\
2018\end{array}$ & 11.4 & 6.1 & 6.0 & 4.2 & 5.4 & 2.7 & 185 \\
\hline Mar -Aug, 2018 & 7.5 & 3.1 & 4.0 & 2.5 & 3.5 & 1.4 & 226 \\
\hline $\begin{array}{l}\text { Sep, } 2018 \text { - Feb } \\
2019\end{array}$ & 7.3 & 3.3 & 4.2 & 2.7 & 3.1 & 1.2 & 315 \\
\hline \multicolumn{8}{|l|}{ Hyderabad } \\
\hline $\begin{array}{l}\text { Sep } 2016 \text { - Feb, } \\
2017\end{array}$ & 5.3 & 2.7 & 3.4 & 2.1 & 1.9 & 1.3 & 172 \\
\hline Mar - Aug, 2017 & 5.8 & 2.7 & 3.7 & 2.0 & 2.1 & 1.3 & 277 \\
\hline $\begin{array}{l}\text { Sep, } 2017 \text {-Feb, } \\
2018\end{array}$ & 6.3 & 2.3 & 3.9 & 1.9 & 2.3 & 1.1 & 615 \\
\hline Mar -Aug, 2018 & 6.7 & 2.7 & 3.8 & 2.2 & 2.9 & 1.2 & 470 \\
\hline $\begin{array}{l}\text { Sep, } 2018 \text { - Feb } \\
2019\end{array}$ & 9.3 & 3.6 & 5.7 & 3.2 & 3.6 & 1.2 & 384 \\
\hline \multicolumn{8}{|l|}{ Male } \\
\hline $\begin{array}{l}\text { Sep } 2016 \text { - Feb, } \\
2017\end{array}$ & 6.2 & 4.7 & 3.7 & 3.0 & 2.5 & 2.3 & 114 \\
\hline Mar - Aug, 2017 & 8.0 & 5.4 & 4.8 & 3.4 & 3.1 & 2.5 & 213 \\
\hline $\begin{array}{l}\text { Sep, } 2017 \text {-Feb, } \\
2018\end{array}$ & 7.9 & 4.5 & 4.6 & 3.0 & 3.2 & 2.2 & 429 \\
\hline Mar -Aug, 2018 & 7.1 & 2.9 & 3.9 & 2.4 & 3.2 & 1.3 & 360 \\
\hline $\begin{array}{l}\text { Sep, } 2018 \text { - Feb } \\
2019\end{array}$ & 8.4 & 3.8 & 5.1 & 3.3 & 3.3 & 1.2 & 387 \\
\hline Female & & & & & & & \\
\hline
\end{tabular}




\begin{tabular}{|c|c|c|c|c|c|c|c|}
\hline \multirow[t]{2}{*}{ Referral cohorts } & \multicolumn{2}{|c|}{$\begin{array}{l}\text { Total follow-up } \\
\text { visits }\end{array}$} & \multicolumn{2}{|c|}{$\begin{array}{l}\text { Follow-up visits at Continuation } \\
\text { phase }\end{array}$} & \multicolumn{2}{|c|}{$\begin{array}{l}\text { Follow-up visits at Intensive } \\
\text { Phase }\end{array}$} & \multirow[t]{2}{*}{$\begin{array}{l}\text { Number of } \\
\text { cases }\end{array}$} \\
\hline & Mean & SD & Mean & SD & Mean & SD & \\
\hline $\begin{array}{l}\text { Sep } 2016 \text { - Feb, } \\
2017\end{array}$ & 6.1 & 3.4 & 3.9 & 2.5 & 2.2 & 1.6 & 89 \\
\hline Mar - Aug, 2017 & 6.5 & 4.4 & 3.9 & 2.7 & 2.6 & 2.2 & 149 \\
\hline $\begin{array}{l}\text { Sep, } 2017-F e b, \\
2018\end{array}$ & 7.0 & 3.7 & 4.1 & 2.4 & 2.8 & 1.9 & 371 \\
\hline Mar-Aug, 2018 & 6.8 & 2.8 & 3.8 & 2.2 & 3.0 & 1.3 & 335 \\
\hline $\begin{array}{l}\text { Sep, } 2018 \text { - Feb } \\
2019\end{array}$ & 8.4 & 3.4 & 4.9 & 2.8 & 3.5 & 1.2 & 312 \\
\hline \multicolumn{8}{|l|}{ Age $<15$ years } \\
\hline $\begin{array}{l}\text { Sep } 2016 \text { - Feb, } \\
2017\end{array}$ & 7.4 & 3.2 & 5.3 & 3.1 & 2.1 & 1.6 & 7 \\
\hline Mar - Aug, 2017 & 6.1 & 4.0 & 3.9 & 2.6 & 2.2 & 1.8 & 19 \\
\hline $\begin{array}{l}\text { Sep, } 2017-F e b, \\
2018\end{array}$ & 6.6 & 3.8 & 4.0 & 2.5 & 2.6 & 1.8 & 43 \\
\hline Mar -Aug, 2018 & 7.1 & 2.7 & 3.8 & 1.9 & 3.3 & 1.2 & 36 \\
\hline $\begin{array}{l}\text { Sep, } 2018 \text { - Feb } \\
2019\end{array}$ & 7.7 & 2.7 & 4.6 & 2.1 & 3.1 & 1.3 & 23 \\
\hline \multicolumn{8}{|l|}{ Age $15-49$ years } \\
\hline $\begin{array}{l}\text { Sep } 2016 \text { - Feb, } \\
2017\end{array}$ & 6.3 & 4.3 & 3.9 & 2.8 & 2.4 & 2.1 & 154 \\
\hline Mar - Aug, 2017 & 6.9 & 4.5 & 4.2 & 2.8 & 2.7 & 2.2 & 263 \\
\hline $\begin{array}{l}\text { Sep, } 2017 \text {-Feb, } \\
2018\end{array}$ & 7.4 & 4.2 & 4.4 & 2.8 & 3.0 & 2.1 & 580 \\
\hline Mar-Aug, 2018 & 7.0 & 2.8 & 3.9 & 2.3 & 3.1 & 1.3 & 529 \\
\hline $\begin{array}{l}\text { Sep, } 2018 \text { - Feb } \\
2019\end{array}$ & 8.4 & 3.7 & 5.0 & 3.1 & 3.4 & 1.2 & 537 \\
\hline \multicolumn{8}{|l|}{ Age $50+$ years } \\
\hline $\begin{array}{l}\text { Sep } 2016 \text { - Feb, } \\
2017\end{array}$ & 5.4 & 3.7 & 3.2 & 2.5 & 2.2 & 1.9 & 42 \\
\hline Mar - Aug, 2017 & 9.1 & 6.4 & 5.4 & 4.2 & 3.7 & 2.8 & 80 \\
\hline $\begin{array}{l}\text { Sep, } 2017-\text {-Feb, } \\
2018\end{array}$ & 7.8 & 4.3 & 4.5 & 2.7 & 3.2 & 2.2 & 177 \\
\hline Mar -Aug, 2018 & 6.6 & 3.0 & 3.6 & 2.5 & 3.0 & 1.2 & 131 \\
\hline $\begin{array}{l}\text { Sep, } 2018-\text { Feb } \\
2019\end{array}$ & 8.4 & 3.7 & 5.1 & 3.1 & 3.3 & 1.2 & 139 \\
\hline Total & 7.5 & 3.9 & 4.4 & 2.8 & 3.1 & 1.8 & 2760 \\
\hline
\end{tabular}

Table 3 provides the percentage of TB patients having successful treatment outcomes according to the various referral cohorts. Overall, we noticed that the percentage of patients who experienced successful treatment outcomes improved over time from 87.1 to $91.3 \%$, with small fluctuations in between. The increase in successful treatment outcomes was highest in Bengaluru and 
among those aged $>50$ years of age. The successful treatment outcomes were consistently higher ( $>90 \%)$ among females than among males for all the cohorts examined. 
Table 3

Successful treatment according to various referral cohorts of patients who are referred and followed-up by the CHW

\section{Total}

Referral cohorts

$\%$ with successful outcomes

Number of cases

Sep 2016 - Feb, 2017

87.1

193

Mar - Aug, 2017

90.3

339

Sep, 2017 -Feb, 2018

89.0

735

Mar -Aug, 2018

90.1

636

Sep, 2018 - Feb 2019

91.3

677

\section{Bengaluru}

Sep 2016 - Feb, 2017

64.5

31

Mar - Aug, 2017

74.7

79

Sep, 2017 -Feb, 2018

70.2

161

Mar -Aug, 2018

79.4

214

Sep, 2018 - Feb 2019

85.8

309

\section{Hyderabad}

Sep 2016 - Feb, 2017

91.4

162

Mar - Aug, 2017

95.0

260

Sep, 2017 -Feb, 2018

94.3

574

Mar-Aug, 2018

95.5

422

Sep, 2018 - Feb 2019

95.9

368

\section{Male}

Sep 2016 - Feb, 2017

81.5

85

Mar - Aug, 2017

87.6

138

Sep, 2017 -Feb, 2018

85.5

343

Mar-Aug, 2018

85.5

305

Sep, 2018 - Feb 2019

87.4

303

\section{Female}

Sep 2016 - Feb, 2017

94.1

108

Mar - Aug, 2017

94.2

201

Sep, 2017 -Feb, 2018

93.0

392

Mar -Aug, 2018

95.1

331

Sep, 2018 - Feb 2019

96.0

374

\section{Age $<15$ years}

Sep 2016 - Feb, 2017

100.0

6

Mar - Aug, 2017

100.0

19 


\begin{tabular}{|c|c|c|}
\hline Referral cohorts & $\%$ with successful outcomes & Number of cases \\
\hline Sep, 2017 -Feb, 2018 & 94.7 & 38 \\
\hline Mar -Aug, 2018 & 94.1 & 34 \\
\hline Sep, 2018 - Feb 2019 & 100.0 & 22 \\
\hline \multicolumn{3}{|l|}{ Age $15-49$ years } \\
\hline Sep 2016 - Feb, 2017 & 91.2 & 147 \\
\hline Mar - Aug, 2017 & 91.1 & 247 \\
\hline Sep, 2017 -Feb, 2018 & 89.2 & 539 \\
\hline Mar -Aug, 2018 & 91.5 & 483 \\
\hline Sep, 2018 - Feb 2019 & 91.5 & 520 \\
\hline \multicolumn{3}{|l|}{ Age 50 + years } \\
\hline Sep 2016 - Feb, 2017 & 70.0 & 40 \\
\hline Mar - Aug, 2017 & 84.9 & 73 \\
\hline Sep, 2017 -Feb, 2018 & 86.7 & 158 \\
\hline Mar -Aug, 2018 & 83.2 & 119 \\
\hline Sep, 2018 - Feb 2019 & 88.9 & 135 \\
\hline Total & 89.9 & 2580 \\
\hline
\end{tabular}

The successful treatment outcomes according to the characteristics of the TB patients and according to the characteristics of follow-up visits for the total cohorts of patients during the reference periods is given in the Table 4 . The overall successful treatment outcome was higher in Hyderabad at $95 \%$ as compared to Bengaluru at $79 \%$. The successful treatment outcome was found to be relatively higher among females, patients who are aged below 15 years, patients who are single, patients who have extra pulmonary TB, patients who were not previously treated for TB, patients whose initial weight was equal to or above a median reference standard (females (38 kg) and males $(43 \mathrm{~kg})$ ) [24], patients who did not miss any doses, and patients who received individual counselling on TB awareness, adherence and nutrition or family level counselling. We noticed a higher proportion of successful treatment outcomes among patients who were visited by $\mathrm{CHWs}$, two or more times in the intensive phase of the treatment. For the follow-up visits during the continuation phase and the total visits, we noticed that as the number of visits increased the successful treatment outcomes also increased.

We noticed that a number of patient as well as follow-up visit characteristics were associated with the successful treatment outcome. Hence, we applied multiple logistic regression models in order to identify any significant improvement in the successful treatment outcome across the referral cohorts when the other potential factors are controlled for. We conducted three separate models while considering the follow-up visits during intensive phase, continuation phase as well as the total visits made to know how controlling for these differs for the successful treatment outcomes across the referral cohorts. 
Table 4

Successful treatment outcome and the unadjusted odds ratio according to various characteristics of the patient who are referred and followed-up by the CHW

\begin{tabular}{|c|c|c|c|c|}
\hline Characteristics & $\%$ of successful outcomes & Unadjusted OR & p-value & Number of cases \\
\hline \multicolumn{5}{|l|}{ Name of district } \\
\hline Bangalore & 79.0 & & & 794 \\
\hline Hyderabad & 94.7 & 4.79 & $<0.001$ & 1786 \\
\hline \multicolumn{5}{|l|}{ Age } \\
\hline$<15$ years & 96.6 & & & 119 \\
\hline $15-49$ years & 90.8 & 0.34 & 0.038 & 1936 \\
\hline $50+$ years & 85.0 & 0.20 & 0.002 & 525 \\
\hline \multicolumn{5}{|l|}{ Gender } \\
\hline Female & 94.5 & & & 1174 \\
\hline Male & 86.0 & 0.35 & $<0.001$ & 1406 \\
\hline \multicolumn{5}{|l|}{ Marital status } \\
\hline Currently married & 88.2 & & & 1605 \\
\hline Marriage dissolved & 87.1 & 0.90 & 0.673 & 170 \\
\hline Single (Never married) & 93.9 & 2.07 & $<0.001$ & 805 \\
\hline \multicolumn{5}{|l|}{ HIV Status } \\
\hline Negative & 88.6 & & & 1919 \\
\hline Positive & 72.0 & 0.33 & 0.014 & 25 \\
\hline Unknown & 94.3 & 2.14 & $<0.001$ & 636 \\
\hline \multicolumn{5}{|l|}{ Type of TB } \\
\hline Extra Pulmonary TB & 94.9 & & & 451 \\
\hline Pulmonary TB & 88.8 & 0.43 & $<0.001$ & 2129 \\
\hline \multicolumn{5}{|c|}{ History of previous TB treatment } \\
\hline No & 90.6 & & & 2331 \\
\hline Yes & 82.7 & 0.49 & $<0.001$ & 249 \\
\hline \multicolumn{5}{|l|}{ Initial weight ${ }^{a}$} \\
\hline Below median value & 86.8 & & & 310 \\
\hline Median value or above & 91.3 & 1.60 & 0.027 & 758 \\
\hline Unknown & 89.8 & 1.34 & 0.117 & 1511 \\
\hline \multicolumn{5}{|c|}{ Patient's Relationship with care supporter } \\
\hline No care supporter & 92.6 & & & 512 \\
\hline Parent & 90.5 & 0.77 & 0.205 & 728 \\
\hline Siblings/Son/Daughter & 85.0 & 0.45 & $<0.001$ & 386 \\
\hline
\end{tabular}




\begin{tabular}{|c|c|c|c|c|}
\hline Characteristics & $\%$ of successful outcomes & Unadjusted OR & p-value & Number of cases \\
\hline Spouse & 88.8 & 0.64 & 0.029 & 678 \\
\hline Others & 92.8 & 1.03 & 0.928 & 276 \\
\hline \multicolumn{5}{|c|}{ Number of visits during CP } \\
\hline$<2$ & 57.5 & & & 348 \\
\hline $2-3$ & 91.0 & 7.51 & $<0.001$ & 446 \\
\hline $4+$ & 95.9 & 17.36 & $<0.001$ & 1786 \\
\hline \multicolumn{5}{|c|}{ Number of visits during IP } \\
\hline$<2$ & 82.3 & & & 417 \\
\hline $2-3$ & 92.2 & 2.55 & $<0.001$ & 1191 \\
\hline $4+$ & 90.3 & 2.02 & $<0.001$ & 972 \\
\hline \multicolumn{5}{|c|}{ Total number of follow-up visits } \\
\hline$<4$ & 63.7 & & & 311 \\
\hline $4-7$ & 91.7 & 6.29 & $<0.001$ & 1022 \\
\hline $8+$ & 94.9 & 10.73 & $<0.001$ & 1247 \\
\hline \multicolumn{5}{|c|}{ Missed any doses } \\
\hline No & 91.2 & & & 2264 \\
\hline Yes & 80.7 & 0.41 & $<0.001$ & 316 \\
\hline \multicolumn{5}{|c|}{ Provided TB awareness counselling } \\
\hline No & 88.6 & & & 2089 \\
\hline Yes & 95.5 & 2.75 & $<0.001$ & 491 \\
\hline \multicolumn{5}{|c|}{ Provided adherence counselling } \\
\hline No & 87.7 & & & 1888 \\
\hline Yes & 96.0 & 3.34 & $<0.001$ & 692 \\
\hline \multicolumn{5}{|c|}{ Provided nutritional counselling/support } \\
\hline No & 88.3 & & & 1960 \\
\hline Yes & 94.8 & 2.43 & $<0.001$ & 620 \\
\hline \multicolumn{5}{|c|}{ Provided family level counselling } \\
\hline No & 89.5 & & & 2357 \\
\hline Yes & 94.2 & 1.90 & 0.029 & 223 \\
\hline Total & 89.9 & & & 2580 \\
\hline \multicolumn{5}{|c|}{ Note: successful outcome include cured and treatment completed TB patients } \\
\hline
\end{tabular}

The first model included the number of visits in the intensive phase as well as all other significant factors as per the backward 
stepwise multiple logistic regression model. This model indicated that the successful treatment outcomes over the different referral cohorts were not significant and thus were not included in the model (Table 5). In other words, we did not notice any independent effect of the referral cohorts on the successful treatment outcomes in the model that considered the follow-up visits in the intensive phase as a control variable. The same model also suggested that successful treatment outcomes were significantly higher in Hyderabad than Bengaluru, patients who were never married, who received more than 2 follow-up visits during the IP visits, whose initial weight is more than the median weight according to sex, who received adherence counselling and who received nutritional counselling and/or nutritional support during the follow-up visits. However, male patients, patients who have pulmonary TB, who are previously treated for TB and who missed any dose are significantly less likely to experience a successful treatment outcome. Though the initial weight and nutritional counselling and/or nutritional support were identified to be a significant factor in the first model. However, in the second and third model its effect was not significant hence excluded in the models that included the follow-up visits during the continuation phase and the total follow-up visits, respectively. 
Table 5

Results of multivariate logistic regression model for successful outcome among TB patient referred and followed-up by CHW

Characteristics

Model 1

AOR $\quad 95 \% \mathrm{Cl}$
Model 2

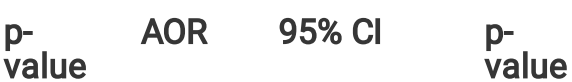

Model 3

AOR $\quad 95 \% \mathrm{Cl} \quad \begin{aligned} & \mathrm{p}- \\ & \text { value }\end{aligned}$

\section{Referral cohort}

Sep 2016 - Feb, $2017^{a}$

Mar - Aug, 2017

Sep, 2017 -Feb, 2018

$1.11 \quad[0.56-$

2.20]

$0.760 \quad 1.16$

[0.61-

0.653

$\begin{array}{ll}0.74 & {[0.40-} \\ & 1.35]\end{array}$

$\begin{array}{lll}0.326 & 0.81 & {[0.45-} \\ & & 1.44]\end{array}$

0.468

Mar -Aug, 2018

$\begin{array}{ll}1.35 & {[0.72-} \\ 2.54]\end{array}$

$\left.\begin{array}{lll}0.346 & 1.25 & {[0.68-} \\ & & 2.28\end{array}\right]$

0.477

Sep, 2018 - Feb 2019

$\begin{array}{ll}2.48 & {[1.28-} \\ & 4.81]\end{array}$

$0.007 \quad 2.21$

[1.154.27]

0.018

\section{Name of district}

Bangalore $^{a}$

Hyderabad

$\begin{array}{lll}5.45 & {[3.75-} & < \\ & 7.92] & 0.001\end{array}$

5.15

[3.43-

7.75]

0.001

6.39

[4.17-

9.78]

$<$

0.001

\section{Gender}

Female $^{a}$

Male

$0.48 \quad[0.35-$

$0.67]$

<.001

0.44

$[0.30-$

$0.62]$

<.001

0.43

$[0.30-$

$0.60]$

$<$

0.001

\section{Marital status}

Currently married ${ }^{a}$

Marriage dissolved

$\begin{array}{lll}0.88 & {[0.51-} & 0.639 \\ 1.51] & \end{array}$

0.89

[0.49-

1.61]

Single

$\begin{array}{lll}1.68 & {[1.14-} & 0.008 \\ & 2.48] & \end{array}$

1.59

$[1.05-$

2.40]

\section{HIV Status}

Negative ${ }^{a}$

Positive

Unknown

$\left.\begin{array}{ll}0.28 & {[0.09-} \\ & 0.85]\end{array}\right] \begin{array}{ll}1.42 & {[0.89-} \\ & 2.28]\end{array}$

0.025

0.30

$[0.10-$

0.029

$2.28]$

0.14

1.21

$[0.77-$

0.418

\section{Type of TB}

Extra Pulmonary $\mathrm{TB}^{\mathrm{a}}$

Pulmonary TB

$\begin{array}{ll}0.65 & {[0.41-} \\ 1.05]\end{array}$

$0.076 \quad 0.50$

$[0.30-$

0.01

0.58

$[0.35-$

$0.96]$

0.034

\section{History of previous TB treatment}

$\mathrm{No}^{\mathrm{a}}$ 


\begin{tabular}{|c|c|c|c|c|c|c|c|c|c|}
\hline \multirow[t]{2}{*}{ Characteristics } & \multicolumn{3}{|c|}{ Model 1} & \multicolumn{3}{|c|}{ Model 2} & \multicolumn{3}{|c|}{ Model 3} \\
\hline & AOR & $95 \% \mathrm{Cl}$ & $\begin{array}{l}\mathrm{p}- \\
\text { value }\end{array}$ & AOR & $95 \% \mathrm{Cl}$ & $\begin{array}{l}\mathrm{p}- \\
\text { value }\end{array}$ & AOR & $95 \% \mathrm{Cl}$ & $\begin{array}{l}\mathrm{p} \text { - } \\
\text { value }\end{array}$ \\
\hline Yes & 0.63 & $\begin{array}{l}{[0.42-} \\
0.97]\end{array}$ & 0.035 & 0.56 & {$[0.35-0.9]$} & 0.016 & 0.58 & $\begin{array}{l}{[0.36-} \\
0.91]\end{array}$ & 0.019 \\
\hline \multicolumn{10}{|c|}{$\begin{array}{l}\text { Patients' Relationship with care } \\
\text { supporter }\end{array}$} \\
\hline \multicolumn{10}{|l|}{ No care supporter ${ }^{a}$} \\
\hline Parent & 1.23 & $\begin{array}{l}{[0.76-} \\
2.01]\end{array}$ & 0.398 & 2.23 & $\begin{array}{l}{[1.31-} \\
3.82]\end{array}$ & 0.003 & 1.48 & $\begin{array}{l}{[0.88-} \\
2.50]\end{array}$ & 0.139 \\
\hline Siblings/Son/Daughter & 0.79 & $\begin{array}{l}{[0.48-} \\
1.29]\end{array}$ & 0.347 & 1.10 & $\begin{array}{l}{[0.63-} \\
1.93]\end{array}$ & 0.729 & 0.94 & $\begin{array}{l}{[0.55-} \\
1.61]\end{array}$ & 0.828 \\
\hline Spouse & 1.45 & $\begin{array}{l}{[0.89-} \\
2.34]\end{array}$ & 0.133 & 1.60 & $\begin{array}{l}{[0.95-} \\
2.68]\end{array}$ & 0.076 & 1.49 & $\begin{array}{l}{[0.89-} \\
2.48]\end{array}$ & 0.126 \\
\hline Others & 1.36 & $\begin{array}{l}{[0.76-} \\
2.45]\end{array}$ & 0.297 & 1.97 & $\begin{array}{l}{[1.03-} \\
3.75]\end{array}$ & 0.041 & 1.74 & $\begin{array}{l}{[0.93-} \\
3.26]\end{array}$ & 0.082 \\
\hline \multicolumn{10}{|c|}{ Number of visits during CP } \\
\hline \multicolumn{10}{|l|}{$<2^{\mathrm{a}}$} \\
\hline $2-3$ & & & & 7.56 & $\begin{array}{l}{[4.86-} \\
11.74]\end{array}$ & $<0.001$ & & & \\
\hline $4+$ & & & & 28.89 & $\begin{array}{l}{[19.54-} \\
42.72]\end{array}$ & $<001$ & & & \\
\hline \multicolumn{10}{|l|}{ Number of visits during IP } \\
\hline \multicolumn{10}{|l|}{$<2^{\mathrm{a}}$} \\
\hline $2-3$ & 2.66 & $\begin{array}{l}{[1.84-} \\
3.84]\end{array}$ & $<001$ & & & & & & \\
\hline $4+$ & 3.85 & $\begin{array}{l}{[2.61-} \\
5.69]\end{array}$ & $<001$ & & & & & & \\
\hline \multicolumn{10}{|c|}{ Total number of follow-up visits } \\
\hline \multicolumn{10}{|l|}{$<4^{\mathrm{a}}$} \\
\hline $4-7$ & & & & & & & 6.48 & $\begin{array}{l}{[4.44-} \\
9.45]\end{array}$ & $\dot{0} 001$ \\
\hline $8+$ & & & & & & & 22.71 & $\begin{array}{l}{[14.71-} \\
35.06]\end{array}$ & $\begin{array}{l}< \\
0.001\end{array}$ \\
\hline \multicolumn{10}{|l|}{ Missed any doses } \\
\hline \multicolumn{10}{|l|}{$\mathrm{No}^{\mathrm{a}}$} \\
\hline Yes & 0.34 & $\begin{array}{l}{[0.23-} \\
0.49]\end{array}$ & $<001$ & 0.23 & $\begin{array}{l}{[0.15-} \\
0.35]\end{array}$ & $<001$ & 0.25 & $\begin{array}{l}{[0.16-} \\
0.37]\end{array}$ & $\begin{array}{l}<.001 \\
0.001\end{array}$ \\
\hline \multicolumn{10}{|c|}{ Provided adherence counselling } \\
\hline \multicolumn{10}{|l|}{$\mathrm{No}^{\mathrm{a}}$} \\
\hline Yes & 2.06 & $\begin{array}{l}{[1.28-} \\
3.31]\end{array}$ & 0.003 & & & & 1.62 & $\begin{array}{l}{[0.99-} \\
2.65]\end{array}$ & 0.057 \\
\hline
\end{tabular}




\begin{tabular}{|c|c|c|c|c|c|c|c|c|c|}
\hline \multirow[t]{2}{*}{ Characteristics } & \multicolumn{3}{|c|}{ Model 1} & \multicolumn{3}{|c|}{ Model 2} & \multicolumn{3}{|c|}{ Model 3} \\
\hline & AOR & $95 \% \mathrm{Cl}$ & $\begin{array}{l}\text { p- } \\
\text { value }\end{array}$ & AOR & $95 \% \mathrm{Cl}$ & $\begin{array}{l}\mathrm{p}- \\
\text { value }\end{array}$ & AOR & $95 \% \mathrm{Cl}$ & $\begin{array}{l}\text { p- } \\
\text { value }\end{array}$ \\
\hline \multicolumn{10}{|l|}{$\begin{array}{l}\text { Provided nutritional } \\
\text { counselling/support }\end{array}$} \\
\hline \multicolumn{10}{|l|}{$\mathrm{No}^{\mathrm{a}}$} \\
\hline Yes & 1.88 & $\begin{array}{l}{[1.19-} \\
2.98]\end{array}$ & 0.007 & & & & & & \\
\hline \multicolumn{10}{|l|}{ Initial weight } \\
\hline \multicolumn{10}{|l|}{ Below median value ${ }^{a}$} \\
\hline Median value or above & 1.63 & $\begin{array}{l}{[1.03-} \\
2.59]\end{array}$ & 0.038 & & & & & & \\
\hline Unknown & 1.06 & $\begin{array}{l}{[0.67-} \\
1.67]\end{array}$ & 0.814 & & & & & & \\
\hline \multicolumn{10}{|c|}{$\begin{array}{l}\text { Model } 1 \text { is based on the stepwise backward deletion multivariate logistic regression with } 0.10 p \text {-value as a group as a whole } \\
\text { and included the number of follow-up visits during the intensive phase and other characteristics of the patient }\end{array}$} \\
\hline \multicolumn{10}{|c|}{$\begin{array}{l}\text { Model } 2 \text { is based on the stepwise backward deletion multivariate logistic regression with } 0.10 \mathrm{p} \text {-value as a group as a whole } \\
\text { and included the number of follow-up visits during the continuation phase and other characteristics of the patient }\end{array}$} \\
\hline \multicolumn{10}{|c|}{$\begin{array}{l}\text { Model } 3 \text { is based on the stepwise backward deletion multivariate logistic regression with } 0.10 \mathrm{p} \text {-value as a group as whole } \\
\text { and included the number of follow-up visits and other characteristics of the patient }\end{array}$} \\
\hline
\end{tabular}

In the second model, we found significantly higher successful treatment outcome for patients whose care supporter is parent, and other relatives; such as uncle/aunt, in-laws, grand-parents or friends as compared to patients who are under self-care. The result also suggests that the likelihood of successful treatment outcome is significantly higher as the number of follow-up visits during the continuation phase are increased to 2 or more visits. Also, we noticed that HIV positive TB patients were found to have significantly lower successful treatment outcomes.

According to the third model, the chance of successful treatment increases as the total number of follow-up visits is 4 or more as compared to patients who received less than 4 follow-up visits totally. The results from the second and third model indicated that the cohorts of patients who were referred during September 2018 and February 2019 were found to have significantly higher likelihood of completing the treatment successfully as compared to the patients who were referred during September 2016 and February 2017.

The common variables that we identified to have significantly influenced the successful treatment outcome in all the three models were sex of the patient, place (city) of patient, type of TB, status of previous TB treatment and the experience of missed doses. The findings from the analysis also indicate that the $\mathrm{CHW}$ approach to improve the TB care cascade may not yield immediate results and may require minimum of two years of implementation to get significant positive results.

\section{Discussion}

The THALI project recruited and trained CHWs to engage with communities for TB detection through the 'screening pathway' and for treatment follow-up and support. Findings demonstrated that this is a fairly effective mode of identifying TB within vulnerable communities and ensuring treatment adherence in order to improve TB treatment outcomes. The $\mathrm{CHWs}$ require some time to establish credibility within the community and with the health services. This is evident in that for the initial few months the proportion of referred persons undergoing the TB testing was comparatively lower. Evidence from the analysis indicates that systematic and consistent community outreach activities by the trained CHWs steadily improved TB detection and TB treatment outcomes. Community engagement through $\mathrm{CHWs}$ is a slow process, but it plugged gaps in the continuum of care cascade, 
resulting in improved TB case detection and TB treatment outcomes over time in the urban slums of both metro cities. The results also substantiate the fact that specific sub-populations need more attention to enhance successful treatment outcomes [21]. The reasons for a difference in TB case detection and successful treatment outcomes between the two cities needs further exploration.

According to the recent TB India report, the percentage of recently diagnosed TB patients put on treatment was $94 \%$ in Karnataka and $97 \%$ in Telangana, which is lower than the percentage of patients put on treatment from the $\mathrm{CHW}$ referred patients in these two cities [25]. The overall successful treatment outcomes rate was $87 \%$ in Karnataka (re-calculation after removing the patients who are not evaluated and whose regimen changed from the calculation) which is close to the rate identified for the latest cohort of TB patients referred in Bengaluru (86\%). However, the success rate was $92 \%$ in Telangana which is slightly lower than the rate identified for the latest cohorts of TB patients referred and cared by CHWs in Hyderabad (96\%). The CHW approach was able to retain a high proportion of patients on treatment and improve successful TB treatment outcomes as compared to the state average. Additionally, the increase is substantial in Bengaluru and among those aged above 50 years of age.

A meta-analysis estimated that $39 \%(95 \% \mathrm{Cl}: 30 \%-49 \%)$ of presumptive TB adults had not seen any medical provider [26]. Thus it is possible that many people with presumptive TB would not seek care at a health facility and this points towards the need for active case finding through the 'screening pathways' adopted in urban slums in these two cities [27]. A recently published study from Myanmar reported similar treatment outcomes between cases identified actively and passively [28]. Similarly, another study in India did not find any significant difference in the TB treatment outcomes of people detected through active case finding as compared to passive case finding among marginalized and vulnerable populations [29]. Previous studies had the limitation of not adjusting for the potential baseline characteristics and also the follow-up characteristics [28].

Though we identified advantages in employing trained CHWs in improving the care cascade, the approach has few limitations. For example, the result indicated $30 \%$ of TB diagnosed individuals were not registered for PCS, higher in Hyderabad as compared to Bengaluru, although they were initially referred for TB diagnosis by the CHW. It is possible that a segment of the population will never be willing for individualized follow-up by a CHW. Self-perceived stigma and discrimination could be one of the reasons for this [30-31]. The project encouraged such individuals who were unwilling for individualized follow up, to attend patient support group (PSG) meetings. Unfortunately, we are not able to track what proportion of individuals attended the PSG meetings as this approach was adopted at the end of the project.

It is ideal that follow up must be initiated in the IP of treatment, and needs to be continued into the CP. We notice that 2 or more visits in the IP and 4 and more visits during CP follow up are associated with successful treatment outcomes of more than $90 \%$. It is also possible that death rates are higher in the intensive phase, as a result of which follow up later is not possible. A study conducted in Northwest Ethiopia indicated 57\% of TB deaths occurred during the intensive phase of the treatment, and the median time of death was two months since initiation of the treatment [32]. Though, overall we noticed an increase in the number of follow-up visits over the time period as observed for the various referral cohorts, Bengaluru witnessed a reduction in the number of follow-up visits. This could be the result of an additional number of patients who were to be followed up in Bengaluru in addition to the $\mathrm{CHW}$ referred patients. The program in later stages demanded that $\mathrm{CHWs}$ follow up all patients in their area, irrespective of whether they had initially referred them or not. These patients who were not initially detected as a result of the $\mathrm{CHW}$ efforts are not included in the analysis. However, this increase in the patient load could have increased the burden on the CHWS, thereby reducing the number of follow-up visits, particularly in Bengaluru.

Significant treatment outcome was noticed for patients who had counselling on treatment adherence and on nutrition or nutrition support offered through the $\mathrm{CHWs}$ and also for patients who did not miss any doses. A study from the Cochrane data base suggested that educational or counselling interventions may improve completion of treatment for latent tuberculosis [33]. However, there is insufficient research to determine whether the routine provision of food or energy supplements in addition to standard care results in better TB treatment outcomes, or improved quality of life [34]. Another study found that participants without any missed doses had a higher TB treatment completion rate as compared to participants who missed $10 \%$ or more of prescribed doses [35].

Page 20/24 
The study also demonstrates that a patient centered approach may involve differentiating the intensity and package of care for certain categories of patients [36]. These include patients co-infected with HIV and previously TB treated patients [23]. Special attention may have to be given to those without an adult care-giver [36]. Even after controlling for other potential factors, the successful treatment outcome is significantly higher in Hyderabad than Bengaluru, though we provided similar kind of patient centered approach during the treatment. Further analysis is required to understand the reasons for this difference. Nevertheless, we noticed a significant improvement in the successful treatment outcomes between the first and last referral cohorts as indicated by the adjusted odds ratio.

There are a number of challenges in the study. The proportion of children $<15$ years of age detected with TB is $4.9 \%$ of all TB cases detected by CHWs. This is lower than the national average of 6.3\% [25]. Most parents tend to opt for private sector services for their children's health issues. Our CHW program referrals were limited to the public sector, as we were not able to engage with the private sector for various reasons. However, we notice that the ratio of females to males detected with TB is much higher than national and state averages [25]. The CHW approach may therefore be useful to detect TB among vulnerable women in the community, but may need to be boosted to ensure that men with TB are not missed. Though we adopted a systematic and consistent patient centered approach we found fluctuations in the care cascade. For example, though the testing rate improved over time, we noted that all the persons referred were not tested, all the persons initiated on treatment did not agree for PCS follow-up visits, and the treatment outcomes are not available for all the patients followed-up. The quality of referrals and quality of interactions with the community and or patient may be important to motivate and change behavior [3738]. This is also dependent on the patient load and the area covered by the CHWs. We were not able to examine these aspects as we did not have this data. Also, we did not include the health system level factors including availability, accessibility, facility readiness and quality of TB diagnostic, treatment and care services, which could have influenced the difference observed between Hyderabad and Bengaluru.

\section{Conclusions}

It is evident from the study that community engagement with slum population conducted through $\mathrm{CHW}$ is a feasible approach and augmented the care cascade of TB detection, treatment initiation and successful treatment outcomes over the period. Case detection increased fairly rapidly, but it takes time before treatment outcomes can be improved. It would be useful to estimate the costs of the model for TB patient detection and successful treatment outcomes.

\section{Abbreviations}

AOR: adjusted odds ratio; ATT: anti-tuberculosis treatment; CBNAAT: cartridge based nucleic acid amplification test; CC: community coordinators; $\mathrm{CHW}$ : community health worker; Cl: confidence interval; CMIS: computerized management information system; DBT: Direct Benefit Transfer; DMC: Designated Microscopy Centre; HIV: human immunodeficiency virus; IEC: Information Education and Communication; KHPT: Karnataka Health Promotion Trust; NTEP: National TB Elimination Program; PCS: prevention care and support card; PRAD: Patient Referral and Diagnosis; PSG: Patient Support Group; RNTCP: Revised National Tuberculosis Control Program; STS: senior TB treatment supervisors; TB: tuberculosis; TBAI: TB Alert India; TBHV: TB health visitor; THALI: tuberculosis health action learning initiative; TU: TB Unit; USAID: United States Agency for International Aid.

\section{Declarations}

\section{Ethics approval and consent to participate}

Regulatory approvals for the study were from the respective State Tuberculosis Office and respective local NTEP officials. Ethical approval was obtained from the Institutional Ethics Committee of St John's Medical College and Research Institute, Bengaluru, India. Oral informed consent was taken from all the participants. 


\section{Consent for publication}

Not applicable

\section{Availability of data and materials}

Some restrictions will apply with sharing the data. Data cannot be shared publicly without the approval form the donor agency and the concerned NTEP office.

\section{Competing interests}

The authors have declared that no competing interests exist.

\section{Funding}

This study is made possible by the generous support of the American People through the United States Agency for International Development (USAID). The work described in this article was implemented under the USAID Tuberculosis Health Action Learning Initiative (THALI) Project, managed by Karnataka Health Promotion Trust under the terms of Cooperative Agreement Number AID-386-A-16-00005. Staff of the funding agency critically reviewed the draft and offered comments to improve the paper.

\section{Authors contributions}

RW, RSP, KK, PBS, RD and VP supported the development and finalization of the study tools. PBS, PBS and RD trained the field staff. RD, VP, and PBS coordinated and supervised the data collection and data entry. VP and HLM administratively supported the project and data collection. RSP did the statistical analysis and interpreted the results. RW and RSP wrote the first draft of the manuscript. KK did the final edit. PBS, PBS, RD, AR, RCR, AS, RS, AS, RT, KK, VP and HLM offered comments on the draft and critically revised the manuscript. All authors read and approved the final manuscript.

\section{Acknowledgements}

We express our sincere thanks to our donor - USAID New Delhi, India for funding this study as a part of the larger project namely, Tuberculosis Health Action Learning Initiative (THALI). We are grateful to the government and our civil society partners for their dedicated support. We would like to acknowledge the significant contributions of the CHWs and CCs for the TB detection and treatment outcomes in the project geographies. Finally, we acknowledge all the individual patients and their supportive family members who consented and spent their time to respond to the questions with patience in each follow-up visits.

\section{Disclaimer}

The U.S. Agency for International Development administers the U.S. foreign assistance program providing economic and humanitarian assistance in more than 80 countries worldwide. This presentation is made possible by the generous support of the American people through the United States Agency for International Development (USAID). The contents are the responsibility of the authors and do not necessarily reflect the views of USAID or the United States Government.

\section{References}

1. World Health Organization. Global tuberculosis report 2020. Geneva:; 2020. Licence: CC BY-NC-SA 3.0 IGO.

2. World Health Organization. Global tuberculosis report 2019. Geneva: 2019. Licence: CC BY-NC-SA 3.0 IGO. 
3. Hazarika I. Role of private sector in providing tuberculosis care: Evidence from a population-based survey in India. J Global Infect Dis. 2011;3:19-24.

4. Stop TB. Partnership and World Health Organization, Geneva (2006) Global Plan to Stop TB 2006-2015. Available at: http://www.stoptb.org/assets/documents/global/plan/globalplanfinal.pdf.

5. World Health Organization, Geneva. (2010) Treatment of Tuberculosis: Guidelines-4th edition. Available at: http://apps.who.int/iris/bitstream/10665/44165/1/9789241547833_eng.pdf?ua=1\&ua=1.

6. World Health Organization (WHO). Diagnostic and treatment delay in tuberculosis: An in-depth analysis of the healthseeking behaviour of patients and health system response in seven countries of the Eastern Mediterranean Region, 2006, WHO-EM/TDR/009/E/10.06/1000.

7. Sreeramareddy CT, Panduru KV, Menten J, Van den Ende J. Time delays in diagnosis of pulmonary tuberculosis: a systematic review of literature. BMC Infect Dis. 2009;9(91):15.

8. Sreeramareddy CT, Qin ZZ, Satyanarayana S, Subbaraman R, Pai M. (2014) Delays in diagnosis and treatment of pulmonary tuberculosis in India: a systematic review Int J Tuberc Lung Dis 18(3): 255-266. doi: 10.5588/ijtld.13.0585 PMID: 24670558.

9. Mistry N, Rangan S, Dholakia Y, Lobo E, Shah S, Patil A. Durations and Delays in Care Seeking, Diagnosis and Treatment Initiation in Uncomplicated Pulmonary Tuberculosis Patients in Mumbai, India. PLoS ONE. 2016;11(3):e0152287. doi:10.1371/journal.pone.0152287.

10. Engel N, Ganesh G, Patil M, Yellappa V, Pant Pai N, Vadnais C, et al. Barriers to Point-of-Care Testing in India: Results from Qualitative Research across Different Settings, Users and Major Diseases. PLoS ONE. 2015;10(8):e0135112. doi:10.1371/journal.pone.0135112.

11. World Health Organization. Standards for TB Care in India, Central TB Division, Ministry of Health \& Family Welfare, Government of India. New Delhi: WHO Country Office for India; 2014.

12. Styblo K. Epidemiology of tuberculosis. 2nd edition. The Hague, Royal Netherlands Tuberculosis Association, 1991.

13. Veesa KS, John KR, Moonan PK, Kaliappan SP, Manjunath K, Sagili KD, et al. Diagnostic pathways and direct medical costs incurred by new adult pulmonary tuberculosis patients prior to anti-tuberculosis treatment - Tamil Nadu, India, PLoS ONE 13(2): e0191591. https://doi.org/10.1371/journal.pone.0191591.

14. Mesfin MM, Newell JN, Madeley RJ, Mirzoev TN, Tareke IG, Kifle YT, et al. Cost implications of delays to tuberculosis diagnosis among pulmonary tuberculosis patients in Ethiopia. BMC Public Health. 2010;10:173.

15. World Health Organization. (2013) Systematic screening for active tuberculosis principles and Recommendations, WHO Document Production Services, Geneva, Switzerland WHO/HTM/TB/2013.04.

16. Yen YL, Chen IC, Wu CH, Li WC, Wang CH, Tsai T-C. (2015) Factors associated with delayed recognition of pulmonary tuberculosis in emergency departments in Taiwan. Heart Lung 44(4):353-9. doi: 10.1016/j.hrtlng.2015.03.004 PMID: 25929441.

17. Yimer SA, Norheim G, Namouchi A, Zegeye ED, KinanderW, Tønjum T, et al. (2015) Mycobacterium tuberculosis lineage 7 strains are associated with prolonged patient delay in seeking treatment for pulmonary tuberculosis in Amhara Region, Ethiopia. Journal of Clinical Microbiology 53(4): 1301-9. doi:10.1128/JCM.03566-14 PMID: 25673798.

18. Ministry of Health and Family Welfare. Revised National Tuberculosis Control Programme: National Strategic Plan for Tuberculosis Elimination 2017-2025, March 2017. New Delhi: Central TB Division, Directorate General of Health Services; 2017.

19. Resource Group for Education and Advocacy for Community Health. (2018). Data for Action for Tuberculosis Key and Vulnerable Populations Rapid Assessment Report India (2018), 194, First Floor, Avvai Shanmugam Salai Lane, Off Lloyds Road, Royapettah, Chennai 600014, India.

20. Hossain SMM, Duffield A, Taylor A. An evaluation of the impact of a US\$60 million nutrition programme in Bangladesh. Health Policy Management. 2005;20(1):35-40.

21. Douthwaite $M$, Ward $P$. Increasing contraceptive use in rural Pakistan: an evaluation of the lady health workers programmes. Health Policy Management. 2005;20(2):117-23. 
22. Global Health Workforce Alliance and World Health Organization. (2010) Global Experience of Community Health Workers for Delivery of Health Related Millennium Development Goals: A Systematic Review, Country Case Studies, and Recommendations for Integration into National Health Systems, available at https://www.who.int/workforcealliance/knowledge/resources/chwreport/en/.

23. Reynold Washington RS, Potty A, Rajesham, et al. Is a differentiated care model needed for patients with TB? A cohort analysis of risk factors contributing to unfavourable outcomes among TB patients in two states in South India, BMC Public Health (2020) 20:1158, https://doi.org/10.1186/s12889-020-09257-5.

24. Ministry of Health with Family Welfare. Guidance Document: Nutritional Care and Support for Patients with TB in India. New Delhi: MOHFW; 2017. Central TB Division, Directorate General of Health Services, Nirman Bhavan, New Delhi 110011.

25. Ministry of Health and Family Welfare. India TB report 2020 National Tuberculosis Elimination Programme Annual report, Central TB Division, Nirman Bhawan, New Delhi - 110011.

26. Subbaraman R, Nathavitharana RR, Satyanarayana S, et al. The Tuberculosis Cascade of Care in India's Public Sector: A Systematic Review and Meta-analysis. PLoS Med. 2016;13(10):e1002149.

27. Christian $C$, Burger $C$, Claassens $M$, et al. Patient predictors of health-seeking behaviour for persons coughing for more than two weeks in high-burden tuberculosis communities: the case of the Western Cape, South Africa. BMC Health Serv Res. 2019;19:160.

28. Khaing PS, Kyaw NTT, Satyanarayana S, et al. Treatment outcome of tuberculosis patients detected using accelerated vs. passive case finding in Myanmar. Int J Tuberc Lung Dis. 2018;22:1145-51.

29. Shewade HD, Gupta V, Satyanarayanaa S, Kumar S, Pandey P, Bajpai UN, et al. Activeive versus passive case finding for tuberculosis in marginalised and vulnerable populations in India: comparison of treatment outcomes. Global Health Action. 2019;12:1656451. https://doi.org/10.1080/16549716.2019.1656451.

30. Ngamvithayapong J, Yanai H, Winkvist A, Saisorn S, Diwan V. Feasibility of home-based and health centre-based DOT: perspectives of TB care providers and clients in an HIV-endemic area of Thailand. Int J Tuberc Lung Dis. 2001;5:741-5.

31. Dick J, Schoeman JH. Tuberculosis in the community: 2. The perceptions of members of a tuberculosis health team towards a voluntary health worker programme. Tuber Lung Dis. 1996;77:380-3.

32. Birlie A, Tesfaw G, Dejene T, Woldemichael K. Time to Death and Associated Factors among Tuberculosis Patients in Dangila Woreda, Northwest Ethiopia. PLoS ONE 10(12): e0144244. doi:10.1371/journal.pone.0144244.

33. M'Imunya JM, Kredo T, Volmink J. Patient education and counselling for promoting adherence to treatment for tuberculosis. Cochrane Database of Systematic Reviews 2012, Issue 5. Art. No.: CD006591. DOI: 10.1002/14651858.CD006591.pub2.

34. World Health Organization. Guideline: Nutritional care and support for patients with tuberculosis. Geneva: 2013.

35. Imperial MZ, Nahid P, Phillips PPJ, Davies GR, Fielding K, Hanna D, et al. A patient-level pooled analysis of treatmentshortening regimens for drug-susceptible pulmonary tuberculosis. Nat Med. 2018. November;24(11):1708-15.

36. WHO Regional Office for Europe. A people-centred model of TB care blueprint for EECA countries. First edition, Marmorvej 51 DK-2100 Copenhagen Ø, Denmark, 2017.

37. Viswanathan M, Kraschnewski J, Nishikawa B, Morgan LC, Thieda P, Honeycutt A, Lohr KN, Jonas D. Outcomes of Community Health Worker Interventions. Evidence Report/Technology Assessment No. 181, AHRQ Publication No. $09-E 014$. Rockville: Agency for Healthcare Research and Quality; June 2009.

38. Perales J, Reininger BM, Lee M, Linder HL. Participants' perceptions of interactions with community health workers who promote behavior change: a qualitative characterization from participants with normal, depressive and anxious mood states. Int J Equity Health. 2018;17:19. https://doi.org/10.1186/s12939-018-0729-9. 